\title{
Necesidades de información en pacientes con enfermedades crónicas. ¿Cómo evitar el ruido en la relación médico- paciente?
}

\section{Information needs in patients with chronic diseases. How to avoid noise in the doctor-patient relationship?}

Marc Compte-Pujol. Universidad de Vic-Universidad Central de Cataluña. España. marc.compte@uvic.cat

$[\mathrm{CV}]$ (1) $\mathrm{G}$

Guillem Marca-Francès. Universidad de Vic-Universidad Central de Cataluña. España.

guillem.marca@uvic.cat

$[\underline{\mathrm{CV}}]$ G

Jesica Menéndez-Signorini. Universidad de Vic-Universidad Central de Cataluña. España.

jesicaana.menendez@uvic.cat

$[\mathrm{CV}](1) \mathrm{G}$

Joan Frigola-Reig. Universidad de Vic-Universidad Central de Cataluña. España.

joan.frigola@uvic.cat

$[\underline{\mathrm{CV}}]$ (1) G

Este estudio se ha realizado en el marco del proyecto COMPAX (CSO2015-71419-R) financiado por el Ministerio de Ciencias, Innovación y Universidades del Gobierno de España. A través de este proyecto, se busca la efectividad de una intervención de comunicación para la mejora de la experiencia del paciente durante su estancia en el hospital y se han desarrollado diversos materiales que ayudan a la comprensión y seguimiento de la terapia por parte del paciente.

Cómo citar este artículo / Referencia normalizada

Compte-Pujol, M., Marca-Francès, G., Menéndez-Signorini, J. y Frigola-Reig, J. (2020). Necesidades de información en pacientes con enfermedades crónicas. ¿Cómo evitar el ruido en la relación médico-paciente? Revista Latina de Comunicación Social, 75, 207-223.

https://www.doi.org/10.4185/RLCS-2020-1423

\section{RESUMEN}

En cuestiones de salud, una información adecuada a las necesidades del paciente suele contribuir a una mejor relación con el personal sanitario, en tanto que ambos actores pueden compartir conocimientos y mejorar su comunicación. En esta investigación, hemos realizado un estudio cualitativo fenomenológico, analizando el contenido de experiencias subjetivas explicadas por pacientes crónicos recientemente hospitalizados en la ciudad de Vic (Barcelona), con el objetivo de identificar, desde la perspectiva del paciente, qué mensajes y temáticas son los más importantes para ellos a nivel de información y cómo prefieren que ésta les sea transmitida para evitar el ruido. Para ello, desarrollamos dos focus groups con pacientes y familiares y 15 entrevistas en profundidad a pacientes ingresados en el hospital. En los resultados, los pacientes muestran una clara predilección 
por la información fácilmente entendible y preferiblemente escrita, mientras que a nivel de ruido y problemáticas de comunicación, critican un exceso de comunicación exclusivamente oral e insuficiente por parte de los médicos, algo que les suele generar estrés y les hace sentirse más cercanos a las enfermeras, que resultan más accesibles y comunicativas. Los resultados también apuntan a la apuesta por la co-creación de la información entre profesionales de la salud y los propios pacientes para mejorar futuras relaciones.

PALABRAS CLAVE: enfermedades crónicas; información; relación médico-paciente; ruido.

\begin{abstract}
In terms of healthcare, information adequate to the patient's needs usually contributes to a better relationship with health personnel, since both actors can share knowledge and improve their communication. In this research, we conducted a qualitative phenomenological study, analyzing the content of subjective experiences explained by chronic patients recently hospitalized in the city of Vic (Barcelona). Our aim was identifying, from the patient's perspective, what messages and topics were the most important for them in terms of information and in which way they preferred to receive them in order to avoid noise. To do this, we developed a couple of focus groups with patients and their relatives and 15 in-depth interviews with patients admitted to the hospital. Results show that patients evidence their predilection for easily understandable and preferably written information, while at the level of noise and communication problems, patients criticized an excess of communication exclusively oral and insufficient by doctors, something that often generates stress and makes them feel closer to nurses, who are more accessible and communicative. The results also point to the commitment to the co-creation of information between health professionals and the patients themselves to improve future relationships.
\end{abstract}

KEYWORDS: chronic diseases; doctor-patient relationship; information; noise.

\title{
CONTENIDO
}

1. Introducción. 2. Marco teórico. 3. Objetivos del estudio. 4. Metodología. 5. Limitaciones metodológicas. 6. Resultados. 7. Discusión y conclusiones. 8. Aspectos éticos. 9. Referencias bibliográficas.

Translation of article by Yuhanny Henares (Academic Translation, Universitat de Barcelona).

\section{Introducción}

Los ciudadanos del siglo XXI tenemos una creciente necesidad de información, en especial cuando adoptamos el rol de pacientes. Gracias a Internet, somos cada vez más proactivos y estamos mejor informados en cuestiones de salud, una realidad que suele contribuir a una mejor relación médicopaciente, en tanto que ambos actores pueden compartir conocimientos y mejorar su comunicación, facilitando que el encuentro presencial sea más eficiente (Lupiáñez-Villanueva, 2011).

Para Clarke et al. (2015), Internet es una de las fuentes de información más comunes y preferidas por su facilidad de acceso a la información, si bien, por lo general, los pacientes confían más en sus médicos por su experiencia clínica. En esta misma línea, Marin-Torres et al. (2012) entienden que Internet no reemplaza al médico ni mina su autoridad, sino que contribuye a cambiar el tipo de relación entre ambos, mejorando el nivel de participación del paciente. Y no sólo eso, sino que en menores de 45 años puede incluso producir cambios en la forma de pensar y en los comportamientos de salud (Ibídem). 
En el caso de pacientes crónicos, Internet suele tener un efecto positivo en el proceso de aceptación de la enfermedad y el manejo de la incertidumbre. Además, el ciberespacio permite que el paciente busque la experiencia y vivencia de otros como él y comparta las suyas propias, facilitando el empoderamiento comunitario entre pacientes.

Para Abt Sacks et al. (2013), un paciente bien informado mejora el cumplimiento del tratamiento y programa prescripto proporcionado por el médico y lleva a cabo la autogestión de su enfermedad de forma autónoma y responsable, a la vez que evita riesgos al paciente ante la utilización de información inadecuada y promueve y potencia un proceso de empoderamiento en la salud.

En consonancia, Barbero (2006) destaca que un paciente debidamente informado identifica claramente a su médico de referencia, está más satisfecho de su relación con éste y comprende mucho mejor las explicaciones acerca de su enfermedad y el tratamiento. De hecho, afirma que los pacientes que saben su diagnóstico no sólo no pierden la esperanza, sino que muestran más confianza en el cuidado que reciben.

Por su parte, García-Izquierdo y Muñoz-Miquel (2015) defienden que un paciente bien informado no sólo se sentirá con mayor control de la situación - lo que aliviaría la ansiedad, sobre todo de los estadios iniciales de la enfermedad-, sino que también mostrará una mayor adhesión al plan terapéutico y una mayor participación e implicación en todo el proceso.

Asimismo, los profesionales médicos de todo el mundo apuestan por informar al paciente de un modo cada vez más mesurado y ajustado, contemplando las complejidades del ser humano, las diferencias culturales y procurando equilibrar la difícil relación entre honestidad y esperanza (Núñez Olarte, 2014).

Todo ello en un contexto en el que las entidades prestadoras de servicios sanitarios ponen en marcha modelos organizativos orientados hacia la atención al cliente, en definitiva, al paciente, centrando su preocupación en la satisfacción y respuesta a las expectativas de éstos. Por esta razón es cada vez más importante disponer de instrumentos que exploren los elementos del proceso de comunicación y acceso a la información, así como a la posterior toma de decisiones sobre la salud a partir de la visión del paciente (Simón et al., 2007).

\section{Marco teórico}

\subsection{Caracterización de la relación médico-paciente desde el marco informativo}

La relación médico-paciente puede definirse como "una relación interpersonal con connotaciones éticas, filosóficas y sociológicas, de tipo profesional que sirve de base a la gestión de salud" (Rodríguez Silva, 2006) y puede clasificarse de dos maneras; a) como una cooperación guiada establecida con pacientes que pueden cooperar en los procesos de diagnóstico y tratamiento, o b) como una participación mutua que implica el seguimiento y cumplimiento del tratamiento, como la discusión de las situaciones y actitudes acerca de la causa y evolución de la enfermedad (Ibídem).

Este tipo de relación obliga hacer una referencia sobre la profesión médica en sí misma, que para Carvallo (2005) debería fundamentarse en dos vertientes igualmente importantes: 1) una científicotécnica, mediante la cual el profesional entiende el comportamiento del cuerpo humano y sus patologías; y 2) otra ético-humanista, en la que el profesional debe concebir al paciente en su plenitud y en su profundidad, y en la cual el carácter, la actitud y los valores del profesional de la salud juegan un papel capital para lograrlo. Para ello, Lin et al. (2017) apuestan por la capacitación 
del personal sanitario en comunicación temprana y apropiada para que puedan regular sus emociones y establecer habilidades de comunicación efectivas.

Sin embargo, la realidad no siempre es tan idealizada, y a menudo los pacientes perciben la figura del médico como una autoridad que tiene en sus manos la curación o la mejora del estado de su salud. Por este motivo, se les disculpa que sean distantes, poco claros y que no resuelvan las dudas de los pacientes, a la vez que se acepta con cierta normalidad que el trato con el médico no sea nada afectivo, pero sí efectivo (Simón et al., 2007). Esta situación conlleva que, aunque los pacientes deseen conocer en profundidad el proceso de su patología, no lo tienen asimilado como un derecho, sino más bien suelen creer que el hecho de brindar información e implicarlos en la toma de decisiones no es una obligación profesional, sino más bien una actitud personal voluntaria del facultativo.

La revisión de la literatura nos permite observar que la relación médico-paciente ha evolucionado, no sólo por las innovaciones tecnológicas en el área de los tratamientos y pruebas médicas, sino también en la forma de relacionarse ante un paciente que tiene más posibilidades de indagar sobre su patología a través de fuentes de información externas (Internet, familiares, otros pacientes, etc.).

El propio profesional médico ha transformado la forma de acercarse al paciente, pasando de un modelo de carácter paternalista, donde el médico decidía qué era lo mejor para a un paciente pasivo, a un nuevo paradigma donde el paciente es más activo, autónomo y participa en la toma de decisiones (Carvallo, 2005). En esta nueva realidad, Carvallo (2005), a partir de un estudio previo de Emannuel (1999), destaca tres modelos que se distinguen por resaltar el rol autónomo del paciente. El primero de ellos es el denominado modelo informativo, por el cual el médico es entendido como un técnico experto que brinda la información relevante al paciente y éste último determina la terapéutica a aplicar desde sus valores y principios. El segundo es el modelo interpretativo, según el cual el médico interpreta un rol de consejero al informar al paciente sobre su afección, así como también sobre los riesgos y beneficios de cada intervención, junto a los valores y opinión del paciente. El último y más deseable sería el modelo deliberativo, donde médico y paciente deliberan conjuntamente

qué tipo de valores relacionados con la salud puede y debe buscar el paciente. Aquí el médico

actúa como maestro y amigo, comprometiendo al paciente a un diálogo sobre qué tipo de acción diagnóstica-terapéutica será mejor. Esto es, hay un diálogo de amistad buscando lo mejor para el paciente. (Carvallo, 2005)

De este modelo deliberativo destacamos los conceptos de compromiso y diálogo, como facilitadores o drivers para una relación médico-paciente simétrica que busca la valoración del paciente como un interlocutor válido preparado para la autogestión responsable de su patología y tratamiento. En definitiva, vemos que una correcta comunicación de la información toma relevancia como aspecto fundamental para la autonomía del paciente y ejerce de pilar de una relación médico-paciente más participativa y deliberativa, donde el protagonismo del profesional da paso a un paciente más informado y, consecuentemente, más satisfecho.

Ruiz-Azarola y Perestelo-Pérez (2012) consideran que para lograr una toma de decisiones compartida son de gran utilidad los programas de educación en auto-cuidados, entendidos como una experiencia fundamental para la toma de conciencia del paciente sobre la posibilidad de ser partícipes activos en su proceso de salud, especialmente para pacientes crónicos. Asimismo, estos programas 
educativos influyen positivamente en el aumento de la confianza del paciente para auto-cuidarse, las mejoras en la calidad de vida y en el bienestar psicológico y el incremento de la autoestima y de la actividad (Ibídem).

Sin embargo, Ruiz-Azarola y Perestelo-Pérez (2012) opinan que a algunos profesionales de la salud todavía les cuesta aceptar el creciente empoderamiento de los pacientes, puesto que ello implicaría la pérdida del poder tradicionalmente asignado a los primeros, que serían reacios a delegar una parte del mismo.

\subsection{Ruidos en la comunicación de la información y su efecto en la participación del paciente}

La literatura desvela que en la práctica existen gran cantidad de ruidos y barreras comunicativas que dificultan sobremanera una correcta transmisión de la información entre médico y paciente.

Un primer ruido evidente sería el grado de alfabetización del paciente en temas de salud (Menéndez et al., 2017), es decir, que el receptor puede no compartir el código comunicativo con el emisor.

Un segundo ruido identificado es que la información proporcionada por el médico no siempre es completa y suele enfocarse únicamente en el tratamiento a seguir. Esto genera dudas en los pacientes que, además, consideran que a menudo no comprenden completamente la información que reciben (Barca Fernández et al., 2004).

Otra barrera comunicativa recurrente es la falta de tiempo que el personal sanitario puede dedicar a transmitir la información al paciente, así como el hecho de que el médico no invite al paciente a expresar libremente sus dudas y preocupaciones ni le pregunte si ha entendido o no la información facilitada (Albahiri et al., 2018, Brandes et al., 2015).

Por otro lado, Brandes et al. (2015) identifican una escasa sensación de legitimidad o derecho que el paciente cree tener a expresar sus preocupaciones. Además, el paciente suele tener miedo a preguntar para no deteriorar la relación con el profesional (Ibídem).

En esta línea, para Leydon et al. (2000) existen tres razones fundamentales por las cuales los pacientes no suelen solicitar más información sobre su salud al personal sanitario: 1) la fe en que el médico es un experto y ya sabrá lo que hace; 2) la esperanza, algo básico para aceptar su enfermedad y para evitar información de carácter negativo; y 3) la caridad, vinculada a la aceptación de que la información debe ser compartida con otros pacientes y, por lo tanto, termina por ser inevitablemente limitada debido a los recursos escasos del sistema.

Por su parte, Barca Fernández et al. (2004) consideran que los pacientes no suelen hacer consultas o preguntas debido a tres razones básicas: 1) su pasividad; 2) la confianza ciega en el facultativo equivalente a la "fe" identificada por Leydon et al. (2000)-; y 3) las trabas en la comunicación lenguaje médico específico, interrupciones, falta de empatía-. Por ello, y porque todavía son pocos los pacientes a los que se pide opinión acerca del proceso diagnóstico, los autores consideran que todavía estamos lejos del modelo de relación compartida, en el cual la información fluya bidireccionalmente, permitiendo la toma de decisiones conjunta entre médico y paciente.

\subsection{Necesidades de información por parte de los pacientes}

Hoy en día, identificar las necesidades de información de los pacientes -así como las de los familiares o allegados de los mismos (Padilla-Fortunatti et al., 2018) - se ha convertido en un 
requisito fundamental para los profesionales de la salud, puesto que se ha demostrado que una correcta identificación de dichas necesidades beneficia directamente a las opciones de tratamiento disponibles (Kassianos et al., 2016). Sin embargo, por lo general los pacientes consideran que no se sienten bien informados, que la comunicación es deficiente y que la protección de su intimidad es mejorable (Simón et al., 2007).

Autores como Navarro et al. (2010) o Ahamad et al. (2018) apuestan por identificar las diferencias individuales en dichas necesidades mediante una atención centrada en el paciente y desde un enfoque multidisciplinar. En la misma línea, Hillebregt et al. (2017) defienden que deben identificarse aquellas necesidades críticas para cada perfil del paciente según su contexto social, sus habilidades, su estilo de vida, nivel educativo, percepciones de la enfermedad, antecedentes personales, deseos y capacidades.

Por lo general, Bernad Vallés et al. (2016) sostienen que los pacientes requieren de profesionales con información especializada, competencias científico-técnicas, habilidades comunicativas y tiempo suficiente para dialogar con ellos, conocer sus necesidades de información y sus preferencias. Además, en el caso de enfermos crónicos, los autores defienden la importancia de cumplir con las expectativas de los mismos y tener en cuenta sus preferencias antes de diseñar un tratamiento, en tanto que ello no sólo aumenta su satisfacción con la relación, sino también mejora el efecto de dicho tratamiento.

Más específicamente, Bernad Vallés et al. (2016) detectan que los pacientes manifiestan una preocupación por las habilidades comunicativas de los profesionales, a la vez que solicitan información inteligible y coordinada entre los diferentes profesionales y niveles asistenciales. Además, los pacientes solicitan mayor cantidad de información tanto de actividades preventivas como de la enfermedad, especialmente del proceso diagnóstico y del tratamiento, para que ésta les permita involucrarse en su cuidado y en la toma de decisiones.

De esta manera, la comunicación con el paciente se entiende como algo más que informar como transmisión de información científica objetiva, ya que el ser humano es complejo en sus necesidades de información (Núñez Olarte, 2014). Del mismo modo, Gil Deza et al. (2014) concluyen que, por encima de todo, los pacientes valoran que el personal sanitario muestre conocimiento, humanidad en el trato, honestidad y experiencia.

Para García Milán (2009) la información clínica que se brinda al paciente debe ser real, comprensible para el destinatario y adecuada a sus circunstancias personales y sociales. Mientras que, según Almodóvar et al. (2018), los pacientes necesitas más información objetiva, constructiva y no catastrofista, dada de forma progresiva y adaptada a las características de cada paciente.

Por su parte, Barbero (2006) sostiene que la información se construye, es un proceso y no momentos puntuales, y que la clave de este proceso es el nivel de comunicación afectiva y efectiva que se logra. Además, considera la información válida como:

- Integradora, al tener en cuenta el contexto sociocultural, la familia del paciente y su realidad;

- Dinámica y de doble dirección;

- Secuencial y atenta a la evolución de los que intervienen en el proceso comunicativo;

- Finalista, al considerarse un medio para lograr el control emocional del paciente, la autonomía de las decisiones, la colaboración en el tratamiento y la adaptación a situaciones favorables o adversas. 
Asimismo, Barbero (2006) considera también que un proceso deliberativo que realmente ayude a la toma de decisiones debe adaptarse al otro, a su realidad a sus miedos y asumir el riesgo de tomar esta postura por parte de los profesionales médicos.

Finalmente, observamos que algunas categorías de paciente son especialmente vulnerables con respecto a la obtención y el uso de información de la salud. Concretamente nos referimos a los pacientes de tercera edad, con niveles más bajos de educación, con problemas de salud y que no están interesados y activos en la búsqueda de información. Para estas tipologías de paciente, es fundamental que la información disponible relacionada con la salud sea comprensible y fácilmente accesible, algo que los proveedores de información deben tener en cuenta (Eriksson-Backa et al., 2012).

\section{Objetivos del estudio}

Si bien hemos detectado una abundante literatura especializada en las necesidades de comunicación e información de diferentes tipologías de pacientes, observamos que muchos de ellos apuestan por perfiles de mediana edad y mayoritariamente alfabetizados en temas de salud. En definitiva, suelen estudiar un perfil de edad y de educación que suele estar altamente influenciado por la información de Internet, algo que, como hemos comentado anteriormente, contribuye a una mayor participación de los pacientes y, por ende, a una comunicación más bidireccional con el personal sanitario.

Sin embargo, en la presente investigación hemos optado por analizar una categoría de paciente especialmente vulnerable a nivel de información: un perfil de tercera edad, de entorno rural, con escasa formación y con patología crónica que requiera hospitalización como consecuencia de una reagudización de su enfermedad de base.

En definitiva, pretendemos contribuir a detectar las barreras comunicativas a nivel de acceso y comprensión de la información de esta categoría específica de paciente, así como sus principales necesidades de información, con el objetivo último de vincular aspectos de comunicación a una mejora de la experiencia del paciente, consiguiendo que la información entre facultativos y pacientes no sea únicamente transmitida sino comprendida y retenida, sea puesta en valor para modificar actitudes que supongan mejoras en la adherencia al tratamiento.

De forma específica, los objetivos de esta comunicación son: a) identificar, desde la perspectiva del paciente, qué mensajes y temáticas son los más importantes para ellos a nivel de información y, b) cómo prefieren que ésta les sea transmitida para evitar el ruido.

\section{Metodología}

\subsection{Diseño del estudio}

Este trabajo es parte de una investigación cualitativa más amplia con el objetivo de mejorar la experiencia del paciente interviniendo desde el ámbito de la comunicación en aquellos elementos y momentos que importan a los pacientes crónicos durante su estancia hospitalaria. Para alcanzar este objetivo, el trabajo se ha realizado bajo una metodología cualitativa con enfoque fenomenológico (Palacios-Ceña y Corral Liria, 2010) porque es el más adecuado para conocer los significados construidos por el paciente desde su discurso, como actor (paciente) involucrado en un contexto y situación determinada (hospitalización) analizando el contenido de experiencias subjetivas explicadas por pacientes crónicos recientemente hospitalizados. Lo que distingue a la Fenomenología como metodología es la comprensión y representación de la experiencia del ser social en su propio 
contexto, no sólo entendiéndolo desde su entorno físico y social, sino desde la historia particular y distintiva del sujeto, como así también sus percepciones, aspiraciones y preocupaciones privativas e íntimas de la persona. Para Taylor y Bogdan (1998),

el fenomenólogo quiere entender los fenómenos sociales desde la propia perspectiva del

actor. Examina el modo en que experimenta el mundo. La realidad que importa es lo que las

personas perciben como importante (...) la conducta humana, lo que la gente dice y hace, es

producto del modo en que define su mundo.

La utilización, entonces, de la Fenomenología como perspectiva metodológica es coherente dado que estudia las experiencias vividas en primera persona por el propio individuo. Unas experiencias vividas que, en nuestro caso, hacen referencia a una enfermedad crónica que precisa hospitalización. Desde este punto de vista, la experiencia del paciente puede contextualizarse a través del momento en que ocurre su vivencia, el espacio donde transcurre la misma y la interacción con otros actores involucrados en dicha experiencia.

\subsection{Participantes y selección de la muestra}

La selección de los perfiles de participantes se realizó mediante muestreo teórico intencionado, en función del tipo y estadio de la enfermedad, y se reclutaron entre los meses de abril a junio de 2018. También se utilizó la técnica denominada "bola de nieve", por la cual una persona recomienda a otra interesada en participar del estudio y que cumple los requisitos. Los participantes objeto de este estudio fueron aquellos que procedentes de su domicilio ingresaron en el Consorcio Hospitalario de Vic (CHV) como consecuencia de una reagudización crónica basal. Las enfermedades crónicas objeto del estudio son la Enfermedad Pulmonar Obstructiva Crónica (EPOC) y la Insufiencia Cardíaca Crónica. Adicionalmente, se ha tenido en cuenta la Diabetes Mielítica Typo II al identificarse su relevancia para el episodio de hospitalización, según criterios médicos. Se han excluido de este estudio a aquellos pacientes que padecen aspectos psicomentales que les dificulte la comprensión de las condiciones de su enfermedad y entorno, como así también aquellos en fase de enfermedad avanzada o grave (deceso médicamente estimado en un período igual o menor a 3 meses y/o en cuidados paliativos) y pacientes institucionalizados (pacientes con severo daño neurológico o mental).

La muestra estuvo compuesta por 30 pacientes de edad mayoritariamente avanzada (54 a 86 años), de los cuales el 70,8\% hombres y el 91\% caucásicos. Todos eran residentes en áreas rurales situadas en los alrededores de la ciudad de Vic (Barcelona) y con poco o muy poco conocimiento y utilización de la tecnología. El nivel de conocimiento de su patología o enfermedad es bajo y se encuentran en diferentes estadios de la enfermedad. Como criterio de elegibilidad para participar del estudio, el paciente debía ser admitido en el hospital proveniente de su hogar o Unidad de Emergencias ingresando en sala como consecuencia de una reagudización crónica basal.

\subsection{Técnicas de recogida de datos}

Para recoger los datos de este trabajo se ha llevado a cabo una investigación social al uso. A efectos de ampliar todas las perspectivas del fenómeno se realizó una triangulación entre investigadores, quienes analizaron los datos y las conclusiones obtenidas. Para la obtención de los datos se llevaron a cabo dos focus groups con pacientes y familiares. Para asegurar la participación, los focus groups se llevaron a cabo durante la visita de seguimiento realizada por el médico en la semana posterior a indicarse el alta médica del paciente. 
Además, se realizaron 15 entrevistas en profundidad semi-estructuradas a pacientes durante su estancia en el hospital. Dado que el foco del estudio se encuentra en la experiencia del paciente, es importante mencionar que se entiende como estancia hospitalaria el período de tiempo comprendido entre el momento en que se efectúa la admisión del paciente y su alta o certificación por parte del médico, que determina que el paciente puede abandonar el centro hospitalario. También se realizaron 4 reuniones de co-creación de material con 6 pacientes, 2 médicos, 2 enfermeras, 1 celador y 2 trabajadores del área administrativa del hospital.

Posterior al registro del ingreso, los pacientes fueron consultados si deseaban participar de este estudio y se les explicó detalladamente su contenido y propósito, previo a la firma del consentimiento. En el caso de los focus groups, los pacientes firmaron el consentimiento de participación previo a la realización de la reunión, la cual se llevó a cabo posteriormente a la recepción de la información pertinente al estudio. Los focus groups y las entrevistas se llevaron a cabo entre los meses de abril a junio de 2018.

Antes de comenzar las entrevistas en profundidad y los focus groups y a modo de facilitar la comunicación inicial y crear un clima de confianza, se explicó a los pacientes el objetivo general del estudio y la garantía del anonimato y la confidencialidad en el tratamiento de los datos. Inclusive, se les ofreció la oportunidad de abandonar su participación en cualquier momento de la sesión sin brindar mayores explicaciones de los motivos. Los focus groups tuvieron una duración de 90 minutos cada uno, mientras que las entrevistas en profundidad se desarrollaron en 45 minutos, aproximadamente. Todas las contribuciones aportadas por los pacientes en las 13 entrevistas y 2 focus groups se grabaron en formato de audio $\mathrm{y}$, posteriormente, fueron transcritas literalmente.

\subsection{Análisis de datos}

El procesamiento y análisis de datos siguió los pasos del análisis de contenido cualitativo inductivo. Dado que el abordaje metodológico resalta la calidad y profundidad de los datos sobre la cantidad y, además, para este estudio no se calculó un tamaño muestral, hemos utilizado el principio de Saturación de Datos, dando por finalizado el trabajo de campo y la recolección de los mismos cuando no aparecía ninguno nuevo o relevante. Es decir, cuando ya no se recolecta información significativa a lo ya obtenido.

Toda la información transcrita literal fue revisada por los investigadores, quienes realizaron la codificación y analizaron la introducción de los datos en la herramienta informática de análisis cualitativo Atlas tiTM v7.5.13.

Un análisis y síntesis de resultados previo e inductivo a través de la lectura y codificación de las transcripciones permitió realizar un análisis de contenido temático a partir de las narrativas relevantes puestos en común por los pacientes y sus familiares, como así también una primera aproximación a un marco explicativo de los datos empíricos obtenidos y de las temáticas consideradas importantes. Una segunda relectura permitió la supervisión y segmentación de datos organizándolos en unidades de sentido en las que se incluyeron aquellos fragmentos que comparten el mismo sentido semántico. Para esto, se utilizó el método de comparativas de constantes, a través del cual dos investigadores leen de forma independiente las transcripciones con el objetivo de encontrar unidades de sentido que permitan la indexación de los fragmentos que describen ideas similares. La triangulación de datos garantizó la reflexividad y permitió la comparación de dicha indexación entre los investigadores. Una vez alcanzado un consenso, se procedió a codificar individualmente todas las transcripciones. Los datos obtenidos de ambos focus groups y de las 15 entrevistas en profundidad fueron divididos en dos niveles de estructura de la información obtenida: 
- Nivel 1: segmentación e identificación de las unidades de sentido en las categorías descriptivas

- Nivel 2: construcción de un sistema de temas, incluyendo varias unidades de sentido o categorías.

Los resultados presentados en este trabajo proceden de un análisis secundario del material empírico relativo al ruido y elementos que influyen negativamente en la transmisión de información médicopaciente.

\section{Limitaciones metodológicas}

Se ha optado por la metodología cualitativa ya que nos permite explorar y entender, desde la óptica del paciente, la forma de interpretar este fenómeno estudiado teniendo en cuenta el contexto. Aunque aporta profundidad, su principal limitación se encuentra al tratarse de un estudio exploratorio. Como futuras líneas de investigación proponemos completar los resultados obtenidos y expuestos en este artículo con enfoques metodológicos cualitativos que aporten un mayor nivel de amplitud y generalización.

\section{Resultados}

Del análisis cualitativo de los datos obtenidos se obtuvieron 377 unidades de análisis, las cuales se agruparon en 22 categorías y 6 temas. Para este trabajo nos enfocaremos únicamente en las categorías que hacen referencia a los ruidos que dificultan la información brindada al paciente y a las necesidades de comunicación expresadas por el mismo. Dichas categorías se indican en la siguiente tabla:

Tabla 1. Frecuencia de las categorías y temas.

\begin{tabular}{|c|c|c|c|}
\hline Categorías y temas & Entrevistas & FocusGroup & Total \\
\hline \multicolumn{4}{|c|}{ Contenido de la información } \\
\hline Información proporcionada por el médico & 53 & 16 & 69 \\
\hline Comprensión de la información & 63 & 11 & 74 \\
\hline Falta de información & 10 & 3 & 13 \\
\hline Información sobre la admisión & 37 & 11 & 48 \\
\hline \multicolumn{4}{|c|}{ Relación profesional-paciente } \\
\hline Confía en el profesional & 5 & 4 & 9 \\
\hline Toma de decisiones compartida & 6 & 0 & 6 \\
\hline Cuidado profesional & 26 & 11 & 37 \\
\hline \multicolumn{4}{|c|}{ Pro-actividad del paciente } \\
\hline Contexto familiar & 6 & 3 & 9 \\
\hline Comportamiento y actitud & 19 & 7 & 26 \\
\hline
\end{tabular}

Fuente: elaboración propia.

Asimismo, en la tabla 2 se aporta una muestra de citas literales de los participantes que ilustran los contenidos informativos obtenidos.

Tabla 2. Extractos de las entrevistas y focus groups para cada categoría y tema.

\begin{tabular}{|l|}
\hline \multicolumn{1}{|c|}{ Contenido de la información } \\
\hline Información proporcionada por el médico \\
\hline Una cosa que no está escrita, no la quiero. \\
\hline
\end{tabular}


Me explicaron exactamente el funcionamiento y voy adentro y ya está. Se acabó la historia.

El doctor no es una persona que hable mucho, la verdad. Es un buen médico, pienso, pero no es una persona que se asiente contigo y diga 'mira, te pasará esto, lo otro...'. No. Él dice 'tienes esto, estás muy grave, tenemos que tratar de detenerlo'. Ya está. No dice nada más y a mí sí que me gustaría que... no me saben decir nada... Si que me gustaría que alguien, aunque fuera 'mira, no podemos hacer nada, cuidados paliativos y punto', pero no me lo dice nadie. También me gustaría saberlo.

\section{Comprensión de la información}

Ya está, me dijo eso el médico. Seguramente me dijo eso, asustando a todo el mundo y ya está. Pero yo tirando igual, no pasa nada.

Pero pregunto, porque a veces, bueno, no eres lo suficientemente lista, o por lo que sea no lo entiendes bien. Pues no, prefiero que me lo expliquen bien. O que lo entienda yo bien (...) Explícate bien, que lo entienda yo bien, eso sí.

\section{Falta de información}

Porque pasa el rato... No, no, ellos hacen su trabajo y tú no la entiendes, y te parece que esa chica que de vueltas por allí, aquel médico que da vueltas por allí debería hacer algo. Y ya lo hace, pero tú no lo entiendes.

Yo no quiero que me digan una mentira, quiero que me sean sinceros. Pero me gustaría, sí, que... pero claro, no pueden, fisicamente no pueden.

\section{Relación médico paciente \\ Confía en el profesional}

Sí, te juro que te agradezco lo que has hecho hoy conmigo, porque estaba bastante asustada y, en cambio, me diste una seguridad. Y que la verdad digo 'vale la pena', y que de verdad os he tenido siempre muy bien valorados.

Confias (...) totalmente en el profesional y dices 'no te puede tocar a ti'. Pero claro, después de la segunda operación ya empiezas a dudar un poco de todos. Sin dudar (...) del profesional, ¿eh? Porque él te cuenta la verdad, al menos en mi caso me lo explicaba.

Percepciones de lo que dices 'te ha pasado a ti porque es el médico que no querías'. Eh, yo elegí el médico, aqui no podía reprochar nada... todo lo contrario, es una persona de confianza.

\section{Toma de decisiones compartida}

Van demasiado deprisa, no tienen tiempo de escuchar, no tienen tiempo de escuchar lo que tú quieres decir. Yo a veces quisiera explicar muchas más cosas de las que el médico me pide...

A veces yo pienso que no nos curamos bien porque el médico no tiene tiempo de escuchar lo que nosotros opinamos de la enfermedad.

\section{Cuidado profesional}

Yo en mi caso sólo me faltaría algo... que deberían... a ver, que son muy bonitas, son muy amables, pero a mí siempre me faltaría un punto de cariño, un punto de apoyar, no sé de qué...

Es un poco de todo, es un poco todo... Pero, sobre todo, sobre todo, es un poco más de tiempo que tú puedas explicar tu malestar o tu bienestar, porque a veces también es bueno poder decir 'me encuentro muy bien, he mejorado'. También es bueno poder explicar la alegría, no siempre la tristeza, ¿no?

También es muy bonito poder explicar: 'me encuentro muy bien, he mejorado'. $Y$, se ha perdido aquella relación, se ha perdido, o se está perdiendo, aquella relación médico-paciente, ¿eh?

Hay una parte psicológica que aqui falla, falla mucho, porque no solamente por el enfermo... por el entorno mismo. Hoy mi mujer mismo, que quería más explicaciones y encontraba que, bueno, que quizá no se las daban lo suficiente. Yo quizá soy más tolerante, no es que sea...

\section{Fuente: elaboración propia.}

De las tres categorías seleccionadas para este artículo, la correspondiente a "Contenido de la información" resume los sentimientos y comportamientos con los que paciente debe lidiar en relación a la información que se le proporciona, e incluye cuatro temas propios: a) información sobre la admisión, b) información proporcionada por el médico, c) comprensión de la información y d) falta de información.

Los pacientes fueron consultados si recibieron información sobre la decisión y la razón de ser admitidos en el hospital a través de la unidad de Urgencias. Todos los pacientes manifestaron que fueron informados sobre la admisión y los motivos de dicha admisión: "Abajo (Urgencias) fui bien informada que tenía una infección y tenía que quedarme" [mujer, 70 años]. Sin embargo, si los 
pacientes manifiestan síntomas respiratorios pueden estar confundidos y, algunos de ellos, pueden tener dificultades para recordar la información que les proporcionaron: "no sé si hablamos de ello, pero ya lo sabía porque me sentía enfermo" [hombre, 83 años].

Sobre cómo valoran la información proporcionada por el médico, los pacientes coinciden en que sea el médico quien provee la información y la diagnosis: "el doctor me dio información en detalle" [hombre, 80 años], mientras que otros explicaron que obtuvieron la información en el hospital y no mientras fueron atendidos en los servicios de Atención Primaria: "el doctor me dijo que tenía COPD. El médico de familia no me dijo eso, que tenía COPD, pero me dijo que deje de fumar" [mujer, 54 años]. Adicionalmente, algunos pacientes mencionaron que buscaron por su cuenta información en Internet, aunque no todo lo que encontraron puede ser considerado como información válida o de confianza: "está claro, lo que pasa es que quizás busco mucha información en internet por mí misma (...) en Internet, además sé que no todo lo que encuentro en Internet puede ser de confianza” [hombre, 74 años].

En segundo lugar, la categoría "Relación médico-paciente" incluye tres temas: 1) confianza en el profesional, 2) toma de decisiones compartida y 3) cuidado profesional. En relación al primer tema, las respuestas de los pacientes son unánimes y en todos los casos resaltan la confianza como pilar fundamental de la relación con el médico: "si lo ves de esta manera, yo no entiendo nada, pero tú eres el experto" [hombre, 66 años], "hice lo que me dijeron que hiciera y eso es todo" [hombre, 77 años], "nunca dudo del profesional porque dice la verdad, al menos en mi caso siempre lo hizo conmigo" [hombre, 80 años]. Adicionalmente, los pacientes contestaron negativamente a la pregunta sobre si habían participado en la toma de aquellas decisiones que influenciaran en su bienestar durante la estancia en el hospital: "diría que no" [mujer, 69 años].

Los participantes describen el cuidado profesional de acuerdo a lo que ellos pudieron ver por ellos mismos, como espectadores de lo que ocurría a su alrededor. En este punto, hubo varios comentarios describiendo la frecuencia de visita del profesional, tales como: "el médico vino cada día", siendo el médico el único que explicaba la situación y, además, como el médico y la enfermera interactuaban en sus respectivos roles: "las enfermeras son las que lo hacen, siguen las instrucciones del médico... hacen todo y luego viene el médico con los resultados como si hubiera hecho todo el proceso" [hombre, 76 años].

La tercera y última categoría analizada en este trabajo, la de "Proactividad del paciente", incluye los temas "contexto familiar" y "comportamiento y actitud". En referencia al primer tema, los comentarios de los pacientes estuvieron enfocados en el rol de los cuidadores y, más específicamente, en la responsabilidad de la toma de medicamentos. En este caso, los parientes cuidadores comentaron en muchos casos estar atentos de cualquier cambio en el nombre o número de medicamentos, cuál es su función, la preparación y dosis. También los cuidadores dicen estar a cargo de la preparación de la dosificación y el cumplimiento del tratamiento: "soy la que está a cargo de la medicación. Yo soy, tú (por el paciente) puedes mirar, pero yo pongo la medicación"; mientras que el paciente indica que "ahora yo no me ocupo de nada, ¿no? Como en la última hospitalización ella (por el cuidador) hace todo" [mujer, 70 años].

Respecto a los comportamientos y actitudes los pacientes no realizaron muchos comentarios, aunque el apoyo entre otros pacientes que tienen la misma enfermedad es unánime.

De un modo más general y transversal, también observamos que los pacientes muestran su predilección por la información fácilmente entendible y preferiblemente escrita, mientras que, a nivel de ruido y problemáticas de comunicación, los pacientes suelen criticar un exceso de comunicación exclusivamente oral e insuficiente por parte de los médicos, algo que les suele 
generar estrés y les hace sentirse más cercanos a las enfermeras, quienes resultan más accesibles y comunicativas.

\section{Discusión y conclusiones}

En este trabajo nos focalizamos en detectar las necesidades de información y los ruidos que evocan en la relación médico-paciente en el contexto específico de pacientes crónicos hospitalizados de tercera edad y con un nivel de alfabetización tecnológica baja, por lo que pueden diferir de otros estudios realizados con muestras de diferentes perfiles de paciente.

De todos modos, observamos varias similitudes con estudios anteriores. La más relevante es el hecho de que la mayoría de los pacientes suele necesitar mayor nivel de información que el realmente recibe, si bien, por distintos motivos, no se atreven a solicitarla (Albahiri et al., 2018; Barca Fernández et al., 2004; Brandes et al., 2015; Leydon et al., 2000).

Destacamos también una clara predilección de los pacientes por la información escrita frente a la oral, unos resultados que coinciden con los de García-Izquierdo y Muñoz-Miquel (2015), quienes resaltan que la información escrita garantiza al paciente que la información que reciba sea entendible y actualizada, a la vez que le brinda seguridad sobre el tratamiento propuesto.

En relación a quién provee la información y cómo debería ser el mejor modo de acceder a ella, los pacientes entienden que el médico es el responsable de informar sobre su estado de salud, en detrimento de otras fuentes como Internet. En este sentido, nuestro estudio concuerda con la visión de Clarke et al. (2015) y Marin-Torres et al. (2012).

Nuestro estudio demuestra también que la presión del tiempo es un factor clave que afecta negativamente no sólo al acceso y comprensión de la información, sino a la misma relación médicopaciente, tal y como ya indicaban anteriormente Albahiri et al. (2018), Bernad Vallés et al. (2016) o Brandes et al. (2015), entre otros.

Se observa, además, que para los pacientes es sumamente importante que los médicos puedan establecer con ellos un diálogo simétrico y bidireccional. Reclaman ser vistos como un interlocutor válido del proceso y evidencian que, pese a su deseo de mayor participación (Bernad Valles et al., 2016), sigue predominando un modelo paternalista en la relación médico-paciente (Carvallo, 2005).

Esta falta de participación activa del paciente trae como consecuencia uno de los ruidos más dañinos en la relación: no reconocer el valor del paciente como ser humano y, además, añade la pérdida de respeto y autoridad hacia el profesional. Una realidad que conlleva, por ejemplo, que los pacientes manifiesten abiertamente tener mayor y mejor acceso a las enfermeras que a los médicos, con las cuales construyen una relación de confianza y apertura que les permite comentar temas que van más allá de su enfermedad. En este sentido, detectamos que las enfermeras son aquellos profesionales en quienes los pacientes depositan mayoritariamente sus dudas.

Asimismo, destacamos que los pacientes consideran especialmente importante establecer una atmósfera privada durante las visitas, intentando minimizar todos aquellos ruidos externos que puedan afectarla, por ejemplo, evitando interrupciones, ya sea por personal no sanitario, o bien, por los propios familiares.

Sin embargo, al igual que Kassianos et al. (2016), observamos también la necesidad de incluir a los familiares más cercanos de un modo positivo y activo, en tanto que ayuda a minimizar las incertidumbres y los ruidos en la información proporcionada al paciente. 
Finalmente, como recomendaciones, creemos que es necesario que el médico apropie su vocabulario y enfoque su discurso al contexto y nivel cultural de sus pacientes. Es por ello importante evitar todo aquel tipo de lenguaje excesivamente técnico que dificulte la comprensión por parte del paciente. Esta situación trae como ruido la duda, el miedo y la incertidumbre al paciente. Sin tener en cuenta el contexto cultural y social del paciente, el médico caerá -una vez más- en aquellos ruidos que entorpecen la comunicación entendida como un proceso colectivo y mutuo.

\section{Aspectos éticos}

El estudio fue aprobado por el Comité de Ética de Investigación Clínica de la Fundación de Osona para la investigación y educación sanitaria. La participación fue voluntaria y los participantes fueron informados de forma verbal y escrita sobre los objetivos y contenidos del estudio. Las transcripciones de las entrevistas y los focus groups se analizaron de forma anónima para preservar la identidad de los participantes.

\section{Referencias bibliográficas}

Abt Sacks, A.; Pablo Hernando, S.; Serrano Aguilar, P.; Fernández Vega, E. y Martín Fernández, R. (2013). Necesidades de información y uso de Internet en pacientes con cáncer de mama en España. Gaceta Sanitaria, 27(3), 241-247.

Ahamad, A; Wallner, P.; Salenius, S.; Ross, R. \& Fernández, E. (2018). Information needs expressed during patient-oriented oncology consultations: Quantity, variation, and barriers. Journal of Cancer Education, In press, 1-10.

Albahri, A. H.; Abushibs; A. S. \& Abushibs, N. S. (2018). Barriers to effective communication between family physicians and patients in walk-in centre setting in Dubai: a cross-sectional survey. BMC Health Services Research, 18(637). https://doi.org/10.1186/s12913-018-3457-3

Almodóvar, R.; Gratacós, J. y Zarco, P. (2018). Necesidades informativas de los pacientes con espondiloartritis sobre su enfermedad. Reumatología Clínica, 14(6).

https://doi.org/10.1016/j.reuma.2017.02.004

Barbero, J. (2006). El derecho del paciente a la información: el arte de comunicar. Anales del Sistema Sanitario de Navarra, 29(S3), 19-27.

Barca Fernández, I.; Parejo Miguez, R.; Gutiérrez Martín, P.; Fernández Alarcón, F.; Alejandre Lázaro, G. y López de Castro, F. (2004). La información al paciente y su participación en la toma de decisiones clínicas. Atención Primaria, 33(7), 361-367.

Bernad Vallés, M.; Maderuelo Fernández, J. A. y Moreno González, P. (2016). Necesidades de información sobre salud y enfermedad de usuarios de servicios sanitarios de atención primaria de Salamanca. Atención Primaria, 48(1), 15-24.

Brandes, K.; Linn, A. J.; Smit, E .G. \& van Weert, J. C. M. (2015). Communication study: Patients' reports of barriers to expressing concerns during cancer consultations. Patient Education and Counseling, 98(3), 317-322.

Carvallo, A. (2005). Médicos y profesionalismo. Pacientes e información. Revista Médica de Chile, $113,253-258$. 
Clarke M. A.; Moore, J. L.; Steege, L. M.; Koopman, R. J.; Belden, J. L.; Canfield, S. M.; Meadows, S. E.; Elliott, S. G., Kim, M. S. (2015). Health information needs, sources, and barriers of primary care patients to achieve patient-centered care: A literature review. Health Informatics Journal, $22(4), 1-25$.

Eriksson-Backa, K.; Stefan, E.; Raimo, N. \& Huotari Maija-Leena, A. (2012). Health information literacy in everyday life: A study of Finns aged 65-79 years. Health Informatics Journal, 18(2), 83-94.

Gil Deza, E.; Gercovich, D.; Morgenfeld, E.; Tognelli, F.; Martín Reinas, G., López, P., Montiel, M.; García Gerardi, C.; Rivarola, E. y Gercovich, F. G. (2014). Evaluación de las necesidades de los pacientes respecto a la información y los cuidados médicos tras el diagnóstico de cáncer. Psicooncología, 11(2-3), 389-401.

García-Izquierdo, I. y Muñoz-Miquel, A. (2015), Los folletos de información oncológica en contextos hospitalarios: la perspectiva de pacientes y profesionales sanitarios, Panace@, 16(4), 225-231.

García Millán, A. (2009). La información al paciente como pieza clave de la calidad asistencial. Revista Clínica de Medicina Familiar, 2(6), 275-279.

Hillebregt, C. F.; Vlonk, A. J.; Bruijnzeels, M. A.; van Schayck, O. C. y Chavannes, N. H. (2017). Barriers and facilitators influencing self-management among COPD patients: a mixed methods exploration in primary and affiliated specialist care. International Journal of Chronic Obstructive Pulmonary Disease, 12, 123-133.

Kassianos, A.; Raats, M. \& Gage, H. (2016). An exploratory study on the information needs of prostate cancer patients and their partners. Health Psychology Research, 4, 19-25.

Leydon, G. M.; Boulton, M.; Moynihan, C.; Jones, A.; Mossman, J.; Boudioni, M. \& McPherson, K. (2000). Cancer patients' information needs and information seeking behavior: in depth interview study. The BMJ, 320, 909-913.

Lin, M. F.; Hsu, W. S.; Huang, M. C.; Su, Y. H.; Crawford, P. \& Tang, C. C. (2017). "I couldn't even talk to the patient": Barriers to communicating with cancer patients as perceived by nursing students. Journal of Cancer Care, 26(4). https://doi.org/10.1111/ecc.12648

Lupiáñez-Villanueva, F. (2011). Salud e internet: más allá de la calidad de la información. Revista Española de Cardiología, 64(10), 849-850.

Marin-Torres, V.; Valverde Aliaga, J.; Sánchez Mrió, I.; Sáenz del Castillo, M. I.; Polentinos-Castro, E. y Garrido Barral, A. (2012). Internet como fuente de información sobre salud en pacientes de atención primaria y su influencia en la relación médico-paciente. Atención Primaria, 45(1), 4653.

Menéndez, M. E.; van Hoom, B. T.; Mackert, M.; Donovan, E. E.; Chen, N. C. \& Ring, D. (2017). Patients with limited health literacy ask fewer questions during office visits with hand surgeons. Clinical Orthopaedics and Related Research, 475(5), 1291-1297. 
Navarro, E.; Limonero, J. T.; Maté, J. y Gómez-Romero, M. J. (2010). Necesidades de comunicación e información en el paciente oncológico superviviente. Psicooncología, 7(1), 127-141.

Núñez Olarte, J. M. (2014). Información al paciente en situación terminal: diferencias entre las publicaciones anglosajones y la experiencia diaria en España. Medicina Paliativa, 21(3), 113-120.

Padilla-Fortunatti, C.; Rojas-Silva, N.; Amthauer-Rojas, M. y Molina-Muñoz, Y. (2018), Necesidades de los familiares de pacientes críticos en un hospital académico de Chile, Enfermería Intensiva, 29(1), 32-40.

Palacios-Ceña, D. y Corral Liria, I. (2010). Fundamentos y desarrollo de un protocolo de investigación fenomenológica en enfermería. Enfermería Intensiva, 21(2), 51-92.

Rodríguez Silva, H. (2006). La relación médico-paciente. Revista Cubana de Salud Pública, 32(4), $1-4$.

Ruiz-Azarola, A. y Perestelo-Pérez, L. (2012). Participación ciudadana en salud: formación y toma de decisiones compartida. Informe SESPAS 2012. Gaceta Sanitaria, 26(S1), 158-161.

Simón, P.; Barrio, I. M.; Sánchez, C. M.; Tamayo, M. I.; Molina, A.; Suess, A. y Jiménez, J. M. (2007). Satisfacción de los pacientes con el proceso de información, consentimiento y toma de decisiones durante la hospitalización, Anales del Sistema Sanitario de Navarra, 30(2), 191-198.

Taylor, S. J. y Bogdan, R. (1998). Introducción a los métodos cualitativos de investigación: la búsqueda de significados. Paidós.

\section{AUTORES:}

\section{Marc Compte-Pujol}

Universidad de Vic-Universidad Central de Cataluña. España.

Doctor en Comunicación y master universitario en Estrategia y Creatividad Publicitarias por la Universidad Ramón Llull (URL) de Barcelona (España), licenciado en Comunicación Audiovisual por la Universidad Pompeu Fabra (UPF) y master en Dirección Cinematográfica por ESCAC (Universidad de Barcelona-UB). Imparte docencia sobre Comunicación Audiovisual, Publicidad y Relaciones Públicas como profesor agregado en la Facultad de Empresa y Comunicación de la Universidad de Vic-Universidad Central de Catalunya (UVIC-UCC). Es coordinador del Master Universitario en Marketing Estratégico e Innovación de la UVic-UCC y miembro del Grupo de Investigación Learning, Media \& Social Interactions (LMI) de la UVic-UCC, grupo consolidado y reconocido por la Generalitat de Catalunya (2017 SGR379). Ha publicado diversos artículos en revistas académicas nacionales e internacionales. Sus principales líneas de investigación se centran en las estrategias de comunicación, el place branding, la función del dircom y la enseñanza universitaria de grado y postgrado sobre relaciones públicas.

marc.compte@uvic.cat

\section{Índice H: 5}

Orcid ID: https://orcid.org/0000-0002-6694-2485

\section{Google Scholar:}

https://scholar.google.nl/citations?hl=en\&authorid=15998701968926261269\&user=ikmknx8AAAAJ 


\section{Guillem Marca-Francès}

Universidad de Vic-Universidad Central de Cataluña. España.

Doctor en Comunicación por la Universidad Rovira y Virgili (URV); Licenciado en Bellas Artes por la Universidad de Barcelona (UB) y en Publicidad y Relaciones Públicas por la Universidad Ramón Llull (URL). Profesor Agregado de la Facultad de Empresa y Comunicación (FEC) de la Universidad de Vic - Universidad Central de Catalunya (UVic-UCC). Miembro del Grupo de Investigación Learning, Media \& Social Interactions (2017SGR379). Su investigación está centrada en las relaciones públicas y la comunicación en el ámbito de la salud.

guillem.marca@uvic.cat

Indice $\mathbf{H}: 8$

Orcid ID: https://orcid.org/0000-0002-1586-8625

Google Scholar: https://scholar.google.es/citations?user=v6hT7SIAAAAJ\&hl=ca

\section{Jesica Menéndez-Signorini}

Universidad de Vic-Universidad Central de Cataluña. España.

Doctora en Comunicación Audiovisual y Publicidad por la Universidad Autónoma de Barcelona (UAB). Profesora Asociada del Departamento de Comunicación de la Facultad de Empresa y Comunicación (FEC) de la Universidad de Vic - Universidad Central de Catalunya (UVic-UCC). Profesora Asociada del Departamento de Comunicación Audiovisual y Publicidad de la Universidad Autónoma de Barcelona (UAB) y colaboradora, en dicha casa de estudios, en la coordinación de estudios de tercer ciclo en marketing y comunicación. Miembro del Grupo de Investigación Learning, Media \& Social Interactions (2017SGR379).

jesicaana.menendez@uvic.cat

Orcid ID: https://orcid.org/0000-0002-4833-5956

\section{Google Scholar:}

https://scholar.google.com/citations?hl=es\&user=dPwa1ZsAAAAJ\&view op=list_works\&gmla=AJ sN-F4xEIDpWEj0fBDYCZG7 152wKvs4KQYwS_rZ8349MJPdjZstJlEJiVUHcwBaHXrvU65ZUmkYVfHVoZ_sCEc1btEZcqZ62C41MVo 15H7hMo8rYZ13U

\section{Joan Frigola-Reig}

Universidad de Vic-Universidad Central de Cataluña. España.

Doctor en Comunicación por la Universidad de Barcelona (UB). Profesor Agregado del Departamento de Comunicación de la Facultad de Empresa y Comunicación (FEC) de la Universidad de Vic - Universidad Central de Catalunya (UVic-UCC). Coordinador del Grupo de Investigación Learning, Media \& Social Interactions (LMI) de la UVic-UCC, grupo consolidado y reconocido por la Generalitat de Catalunya (2017 SGR379), especializado en educación, comunicación y acción social. Forma parte del comité científico de revistas científicas como XPA \& Health Communication. joan.frigola@,uvic.cat

\section{Índice $\mathrm{H}: 2$}

Orcid ID: https://orcid.org/0000-0003-4195-1104

Google Scholar: https://scholar.google.es/citations?user=K4zqrqkAAAAJ\&hl=ca 